\title{
Divergência genética entre cinco cultivares de mamoneira
}

\author{
Henrique Fortes Bahia(1), Simone Alves Silva(1), Luzimar Gonzaga Fernandez ${ }^{(2)}$, Carlos Alberto da Silva Ledo ${ }^{(3)}$ \\ e Ricardo Franco Cunha Moreira(1)
}

\begin{abstract}
(1)Universidade Federal do Recôncavo Baiano, Centro de Ciências Agrárias, Ambientais e Biológicas, s/no, CEP 44380-000 Cruz das Almas, BA. E-mail: bahiahf@yahoo.com.br, sas@ufba.br, ricardofcmoreira@yahoo.com.br (2)Universidade Federal da Bahia, Instituto de Ciências da Saúde, Departamento de Bio-Função, Avenida Reitor Miguel Calmon, s/noo, Vale do Canela, CEP 40110-100 Salvador, BA. E-mail: luzimar@ucsal.br (3)Embrapa Mandioca e Fruticultura Tropical, Rua Embrapa, s/no, CEP 44380-000 Cruz das Almas, BA. E-mail: ledo@cnpmf.embrapa.br
\end{abstract}

\begin{abstract}
Resumo - O objetivo deste trabalho foi avaliar a divergência genética entre cultivares de mamoneira (Ricinus communis L.), a partir dos caracteres adaptativos, dos componentes de produção e do rendimento. $\mathrm{O}$ experimento em delineamento em blocos ao acaso, com cinco tratamentos e cinco repetições, foi implantado em Latossolo Amarelo em Cruz das Almas, BA. Os dados foram submetidos à análise de variância, ordenados pelo teste de Scott-Knott, a 5\% de probabilidade e, com base na análise multivariada, realizou-se análise de agrupamento e análise dos componentes principais para determinação da divergência genética. Houve formação de três grupos, ou seja, constatou-se a existência de divergência genética entre os genótipos. A cultivar mais divergente foi Mirante 10, e não é recomendada para hibridação em virtude do baixo desempenho apresentado. Há expectativa de combinações promissoras entre Sipeal 28 x BRS 188 Paraguaçu, Sipeal 28 x BRS 149 Nordestina, EBDA MPA 17 x BRS 188 Paraguaçu e EBDA MPA 17 x BRS 149 Nordestina, em virtude da maior dissimilaridade apresentada e do melhor desempenho médio desses genótipos.
\end{abstract}

Termos para indexação: Ricinus communis, análise multivariada, dissimilaridade, melhoramento genético.

\section{Genetic divergence in five castor bean cultivars}

\begin{abstract}
The objective of this work was to evaluate the genetic divergence between castor bean (Ricinus communis L.) cultivars, from adaptative traits, yield components and cultivars productivity. The experiment in a randomized blocks design with five treatments and five replications was carried out in a Xanthic Hapludox in Cruz das Almas, BA, Brazil. Data were submitted to the variance analysis and the average values had been commanded according to Scott-Knott test $(\alpha=0.05)$. Based on multivariate analysis, the genetic divergence was determined using cluster analysis and principal components analysis. Tree groups were formed, and the one which comprised the cultivate more divergent, Mirante 10, is not recommended for hybridization due to its low performance. Promising combinations are those ones found between Sipeal 28 x BRS 188 Paraguaçu, Sipeal 28 x BRS 149 Nordestina, EBDA MPA 17 x BRS 188 Paraguaçu and EBDA MPA 17 x BRS 149 Nordestina, due to the dissimilarity and the best average performance of these genotypes.
\end{abstract}

Index terms: Ricinus communis, multivariate analysis, dissimilarity, genetic improvement.

\section{Introdução}

A mamoneira é cultivada tradicionalmente em quase toda a Bahia, principalmente por pequenos agricultores, em sistema de consórcio com milho ou feijão. A planta é de grande importância para o Estado por ser rústica, resistente à seca e geradora de renda e ocupação. Apesar de sua importância socioeconômica, a espécie conta com poucas cultivares melhoradas para o Nordeste, embora possua ampla variabilidade genética e seu melhoramento na Região ocorra desde a década de 1960.

Das cultivares lançadas para a região nordestina, as que mais se destacaram foram a Sipeal 04, a Sipeal 28, a BRS 188 Paraguaçu e a BRS 149 Nordestina. Entretanto, dados da Companhia Nacional de 
Abastecimento (2007) revelam que a produtividade no Estado ainda é extremamente baixa, visto que o rendimento médio nos últimos cinco anos foi de aproximadamente $730 \mathrm{~kg} \mathrm{ha}^{-1}$, bem inferior, por exemplo, ao da Região Sudeste no mesmo período (1.377 kg ha-1).

Genótipos como Mirante 10 e EBDA MPA17 estão sendo avaliados pela Empresa Baiana de Desenvolvimento Agrícola S.A. (EBDA), em parceria com a Embrapa Algodão, apresentando resultados promissores em alguns municípios baianos.

Existem perspectivas para obtenção de cultivares que possam aumentar a produtividade do fruto e o teor de óleo das sementes, e permitir a identificação de fontes de resistência às principais pragas e doenças da cultura, já que sua diversidade é ainda pouco explorada (Carvalho, 2005).

A importância da diversidade genética para o melhoramento reside no fato de que cruzamentos envolvendo genitores geneticamente diferentes são os mais convenientes para produzir alto efeito heterótico além de maior variabilidade genética em gerações segregantes. Esse tipo de estudo viabiliza o conhecimento das melhores combinações híbridas e a formação de populações com ampla base genética, proporcionando maior ganho genético nos sucessivos ciclos de seleção (Rao et al., 1981; Falconer, 1987).

A certificação de variabilidade com base na divergência genética é uma estratégia bastante utilizada em um programa de melhoramento. Entretanto, a avaliação da divergência genética como critério de escolha de genitores nos programas de melhoramento genético da mamoneira tem sido pouco realizada. Como exemplo, citam-se os trabalhos de Figueiredo Neto et al. (2004) e Costa et al. (2006).

A avaliação da dissimilaridade ou similaridade é realizada por análises biométricas, com base na quantificação da heterose, ou por processos baseados nas diferenças morfológicas, fisiológicas e agronômicas dos genitores. Um dos procedimentos mais empregados é a distância $\mathrm{D}^{2}$ de Mahalanobis. A análise dos componentes principais permite identificar caracteres que mais explicam a variação existente. Sua utilização reduz a dimensionalidade do conjunto de variáveis originais com um mínimo de perda de informações, possibilitando aplicar técnicas de agrupamento que permitem visualizar a dispersão gráfica em espaços bidimensional ou tridimensional de fácil interpretação geométrica (Cruz, 1990; Cruz \& Carneiro, 2001; Cruz et al., 2004).
A fim de subsidiar o Programa de Melhoramento Genético da Mamoneira para o Recôncavo Baiano, este trabalho objetivou avaliar a divergência genética entre cinco cultivares de mamoneira com base em caracteres adaptativos, componentes de produção e rendimento.

\section{Material e Métodos}

O trabalho foi realizado no Centro de Ciências Agrárias, Ambientais e Biológicas (CCAAB) da Universidade Federal do Recôncavo da Bahia, em Cruz das Almas, BA, entre abril de 2006 e fevereiro de 2007. O Município está situado na região fisiográfica do Recôncavo Baiano, apresentando as coordenadas geográficas de $12^{\circ} 40^{\prime} 19^{\prime \prime} \mathrm{S}, 39^{\circ} 6^{\prime} 23^{\prime \prime} \mathrm{W}$ e altitude média de $220 \mathrm{~m}$. O clima é do tipo subúmido, com pluviosidade média anual de $1.170 \mathrm{~mm}$, com variações entre $900 \mathrm{e}$ $1.300 \mathrm{~mm}$, e os meses de março a agosto são os mais chuvosos, e de setembro a fevereiro, os mais secos. A temperatura média anual é de $24,1^{\circ} \mathrm{C}$ e o solo é classificado como Latossolo Amarelo álico coeso, de textura argilosa e relevo plano (Ribeiro et al., 1995).

As sementes de mamona utilizadas neste trabalho foram obtidas pela EBDA, na Estação Experimental de Iraquara, BA. Estas foram colhidas em janeiro de 2005, beneficiadas e armazenadas em sacos de aniagem na Estação Experimental de Itaberaba, da EBDA, até o plantio no campo experimental do CCAAB.

A correção do solo foi efetuada seguindo recomendações da análise de fertilidade química, sendo aplicados $1.000 \mathrm{~kg} \mathrm{ha}^{-1}$ de calcário dolomítico, $60 \mathrm{~kg} \mathrm{ha}^{-1}$ de $\mathrm{N}$ ( $20 \mathrm{~kg} \mathrm{ha}^{-1}$ no plantio e $40 \mathrm{~kg} \mathrm{ha}^{-1}$ em cobertura), $80 \mathrm{~kg} \mathrm{ha}^{-1}$ de $\mathrm{P}_{2} \mathrm{O}_{5}$ e $40 \mathrm{~kg} \mathrm{ha}^{-1}$ de $\mathrm{K}_{2} \mathrm{O}$. O controle de ervas daninhas foi realizado com capina manual e não foram utilizados inseticidas nem fungicidas para combater a lagarta-rosca (Agrotis ipsilon) e o mofo-cinzento (A. ricini), respectivamente, observados durante a condução do experimento.

O delineamento utilizado foi o de blocos ao acaso, com cinco repetições e cinco tratamentos, constituídos pelas cultivares BRS 149 Nordestina, BRS 188 Paraguaçu, EBDA MPA 17, Mirante 10 e Sipeal 28.

Cada parcela foi composta por cinco fileiras de $12 \mathrm{~m}$, com espaçamento entre fileiras de $3 \mathrm{~m}$. Dentro da fileira, o espaçamento entre covas foi de $1 \mathrm{~m}$, resultando em 12 covas por fileira e 60 covas por parcela. As bordaduras foram constituídas pelas linhas laterais, resultando em 30 covas na área útil da parcela, 
abrangendo as dimensões de 9x10 m. Foram semeadas três sementes por cova e o desbaste foi realizado aos 25 dias após o plantio, deixando uma planta por cova.

Foram analisados os caracteres florescimento do racemo primário (FLO), estande final (EST), número de racemos emitidos por planta (NRE), número de racemos colhidos por planta (NR), número de racemos abortados por planta (NRA), altura de caule (AC), estatura de planta (EP), diâmetro de caule (DC), comprimento médio de internódios do caule (CMIC), número de internódios do caule (NIC), comprimento efetivo (útil) de racemo (CER), comprimento de racemo (CR), comprimento de racemo sem enchimento (CRSE), peso de racemo (PR), peso de frutos por racemo (PFR), peso de sementes por racemo (PSR), número de frutos por racemo (NFR), número de sementes por racemo (NSR), peso de frutos por planta (PFPL), peso de frutos por parcela (PFP), peso de sementes por planta (PSPL), peso de sementes por parcela (PSP) e potencial produtivo de sementes (PP).

Os caracteres FLO, AC, CMIC, NIC, DC, EP, CER e PP foram avaliados de acordo com descritores utilizados pela Embrapa Algodão, definidos por Freire et al. (2001). Para avaliar NR, NRE e o NRA, foram realizadas contagens periódicas durante todo o ciclo da cultura. Nessas aferições, foi considerado racemo abortado o que não apresentou frutos ou encontrava-se deformado ou malformado com número de frutos inferior a três. A fim de estudar o potencial de enchimento do racemo, foram aferidos CR e CRSE. Esses caracteres, mais CER, PR, PFR, PSR e NFR foram realizados nos três primeiros racemos de cada planta e em dez plantas ao acaso, utilizando-se régua e balança digital de precisão. O EST foi determinado contando-se o número de plantas vivas aptas para colheita. Foram realizadas colheitas periódicas a fim de se evitar perdas com deiscência de algumas cultivares, entretanto, a colheita final foi efetuada no mesmo período para todas as cultivares. Os racemos colhidos tiveram os frutos retirados e pesados para determinação do PFP. O PFPL foi determinado pela razão entre o PFP e o EST. Após secagem ao sol em terreiro, as sementes que não foram removidas dos frutos por deiscência, foram retiradas pelas batidas em sacos de aniagem, e as que ainda apresentavam casca aderida, extraídas com alicate de poda manualmente. As sementes foram pesadas para determinação do PSP e, a partir deste, o PP foi mensurado de acordo com o número de covas da área útil e tamanho da área útil. O PSPL foi determinado pela razão entre PSP e EST.

Os dados, inicialmente, foram submetidos à análise de variância para avaliação da presença de variabilidade genética entre as cultivares e, em seguida, os valores médios foram ordenados segundo o teste de Scott-Knott, a 5\% de probabilidade. Nessas análises foi utilizado o SAS (SAS Institute, 2001).

Foram realizadas análises multivariadas utilizando-se técnicas de análise de agrupamento e análise de componentes principais. Para a análise de agrupamento foi utilizada a distância $\mathrm{D}^{2}$ de Mahalanobis como medida de dissimilaridade e no processo de agrupamento, utilizouse o método UPGMA. Essas análises estatísticas foram realizadas com o programa NTSYSPC 2.1 (Rohlf, 2000).

\section{Resultados e Discussão}

$\mathrm{Na}$ análise de variância, houve diferença significativa para a maioria dos caracteres, à exceção do estande final (EST), apresentando variação entre os genótipos, o que indica a necessidade de avaliação das médias separadamente em cada genótipo e sua comparação pelo teste de Scott-Knott. Nesta análise (Tabela 1), observase que as médias somente não diferem significativamente para o estande final, confirmando resultados obtidos pelo teste $\mathrm{F}$, sinalizando, a princípio, existência de divergência genética entre as cultivares. O coeficiente de variação foi satisfatório em todos os caracteres, sugerindo eficiente controle do efeito de ambiente e, conseqüentemente, maior confiabilidade nos dados.

Houve formação de pelo menos dois grupos pelo teste de Scott-Knott em quase todos os caracteres avaliados (Tabela 1). O número de racemos abortados, a altura do caule, o número de internódios do caule e o peso de sementes por racemo foram representados por três grupos. Observa-se ainda a formação de quatro grupos quanto aos caracteres número de frutos por racemo e o número de sementes por racemo. No entanto, assim como observaram Costa et al. (2006), o início do florescimento foi o caráter que apresentou o maior número de grupos, em que verificaram-se cinco grupos, sendo este o maior número possível.

A diversidade constatada entre os genótipos foi observada preliminarmente nos caracteres coloração, tamanho e forma da semente, sendo inclusive, facilmente visualizada nas sementes utilizadas neste trabalho (Figura 1). 
A cultivar Sipeal 28 alcançou os melhores desempenhos em quase todos os componentes de produção: peso de racemo por planta, peso de frutos por racemo, peso de sementes por racemo, peso de frutos por parcela, peso de sementes por planta, peso de sementes por parcela e potencial produtivo de sementes. Essa cultivar apresenta porte médio, caule roxo, sem cera, frutos deiscentes, sementes de cor predominantemente preta, floração do primeiro cacho com 55 dias, teor de óleo nas sementes de 47,3\% e produtividade média em condições de sequeiro de $1.300 \mathrm{~kg} \mathrm{ha}^{-1}$ de bagas (Beltrão, 2004). Segundo Beltrão (2004), a 'Sipeal 28' teve seu melhoramento genético iniciado em Cruz das Almas, na década de

Tabela 1. Médias das características agronômicas de cinco cultivares de mamoneira avaliadas em Cruz das Almas, BA ${ }^{(1)}$.

\begin{tabular}{|c|c|c|c|c|c|c|}
\hline $\begin{array}{l}\text { Características } \\
\text { agronômicas }\end{array}$ & BRS 149 Nordestina & BRS 188 Paraguaçu & EBDA MPA -17 & Mirante 10 & Sipeal 28 & CV (\%) \\
\hline FLO (dias) & $49,60 b$ & $47,00 \mathrm{~d}$ & $48,60 \mathrm{c}$ & $42,20 \mathrm{e}$ & $53,00 \mathrm{a}$ & 1,33 \\
\hline EST & $14,40 \mathrm{a}$ & $18,60 \mathrm{a}$ & $20,20 a$ & $14,60 \mathrm{a}$ & $19,40 \mathrm{a}$ & 26,41 \\
\hline NRE & $23,62 \mathrm{a}$ & $28,10 \mathrm{a}$ & $15,20 b$ & $28,68 \mathrm{a}$ & $19,28 b$ & 18,82 \\
\hline NR & $16,10 \mathrm{a}$ & $18,80 \mathrm{a}$ & $11,36 b$ & $9,56 b$ & $17,44 a$ & 24,81 \\
\hline NRA & $7,52 b$ & $9,30 \mathrm{~b}$ & $3,84 \mathrm{c}$ & $19,12 \mathrm{a}$ & $1,84 \mathrm{c}$ & 22,88 \\
\hline $\mathrm{AC}(\mathrm{cm})$ & $58,76 b$ & $57,42 b$ & $66,65 a$ & $39,42 \mathrm{c}$ & $59,81 b$ & 9,51 \\
\hline $\mathrm{EP}(\mathrm{m})$ & $1,95 b$ & $2,20 \mathrm{a}$ & $2,08 \mathrm{a}$ & $1,79 b$ & $2,34 a$ & 11,33 \\
\hline $\mathrm{DC}(\mathrm{mm})$ & $52,04 a$ & $49,48 \mathrm{a}$ & $49,86 a$ & $38,53 b$ & $50,63 a$ & 10,62 \\
\hline CMIC (cm) & $3,93 b$ & $4,19 b$ & $4,81 \mathrm{a}$ & $4,21 b$ & $4,69 a$ & 10,17 \\
\hline $\mathrm{NIC}$ & $15,76 a$ & $14,14 b$ & $14,26 b$ & $9,96 \mathrm{c}$ & $13,26 b$ & 7,12 \\
\hline CER (cm) & $18,01 \mathrm{a}$ & $14,86 b$ & $18,50 \mathrm{a}$ & $18,14 a$ & $18,12 \mathrm{a}$ & 11,06 \\
\hline $\mathrm{CR}(\mathrm{cm})$ & $24,14 a$ & $19,40 b$ & $25,14 a$ & $23,94 a$ & $23,90 \mathrm{a}$ & 9,32 \\
\hline CRSE (cm) & $6,13 a$ & $4,54 b$ & $6,64 a$ & $5,80 \mathrm{a}$ & $5,78 \mathrm{a}$ & 17,02 \\
\hline PR (g) & $96,16 a$ & $92,77 a$ & $97,50 \mathrm{a}$ & $27,80 \mathrm{~b}$ & $107,44 a$ & 17,21 \\
\hline PFR (g) & $82,80 \mathrm{a}$ & $82,06 a$ & $84,73 a$ & $24,00 \mathrm{~b}$ & $99,11 \mathrm{a}$ & 16,06 \\
\hline PSR (g) & $49,52 b$ & $52,37 \mathrm{~b}$ & $54,44 b$ & $15,64 c$ & $63,66 a$ & 16,61 \\
\hline NFR & $25,48 \mathrm{c}$ & $24,58 \mathrm{c}$ & $36,02 \mathrm{a}$ & $17,26 \mathrm{~d}$ & $28,98 b$ & 14,57 \\
\hline NSR & $71,94 \mathrm{c}$ & $67,58 \mathrm{c}$ & $97,04 a$ & $47,52 d$ & $79,14 b$ & 15,39 \\
\hline PFPL (kg) & $1,32 \mathrm{a}$ & $1,01 \mathrm{a}$ & $0,92 \mathrm{a}$ & $0,45 b$ & $1,20 \mathrm{a}$ & 25,36 \\
\hline PFP (kg) & $18,50 \mathrm{a}$ & $18,02 \mathrm{a}$ & $18,87 \mathrm{a}$ & $6,78 b$ & $22,58 \mathrm{a}$ & 32,24 \\
\hline PSPL (kg) & $0,62 \mathrm{a}$ & $0,57 \mathrm{a}$ & $0,48 \mathrm{a}$ & $0,28 b$ & $0,65 \mathrm{a}$ & 25,09 \\
\hline PSP (kg) & $8,72 \mathrm{a}$ & $10,11 \mathrm{a}$ & $9,78 \mathrm{a}$ & $4,20 \mathrm{~b}$ & $12,13 \mathrm{a}$ & 31,36 \\
\hline $\mathrm{PP}\left(\mathrm{kg} \mathrm{ha}^{-1}\right)$ & $968 \mathrm{a}$ & $1.123 \mathrm{a}$ & $1.086 \mathrm{a}$ & $467 b$ & $1.347 \mathrm{a}$ & 31,36 \\
\hline
\end{tabular}

(1)FLO: florescimento do racemo primário; EST: estande final; NRE: número de racemos emitidos por planta; NR: número de racemos por planta; NRA: número de racemos abortados por planta; AC: altura de caule; EP: estatura de planta; DC: diâmetro de caule; CMIC: comprimento médio de internódios do caule; NIC: número de internódios do caule; CER: comprimento efetivo de racemo; CR: comprimento de racemo; CRSE: comprimento de racemo sem enchimento; PR: peso de racemo; PFR: peso de frutos por racemo; PSR: peso de sementes por racemo; NFR: número de frutos por racemo; NSR: número de sementes por racemo; PFPL: peso de frutos por planta; PFP: peso de frutos por parcela; PSPL: peso de sementes por planta; PSP: peso de sementes por parcela; PP: potencial produtivo de sementes; médias seguidas de letras iguais, na linha, não diferem entre si pelo teste de Scott-Knott, a $5 \%$ de probabilidade.

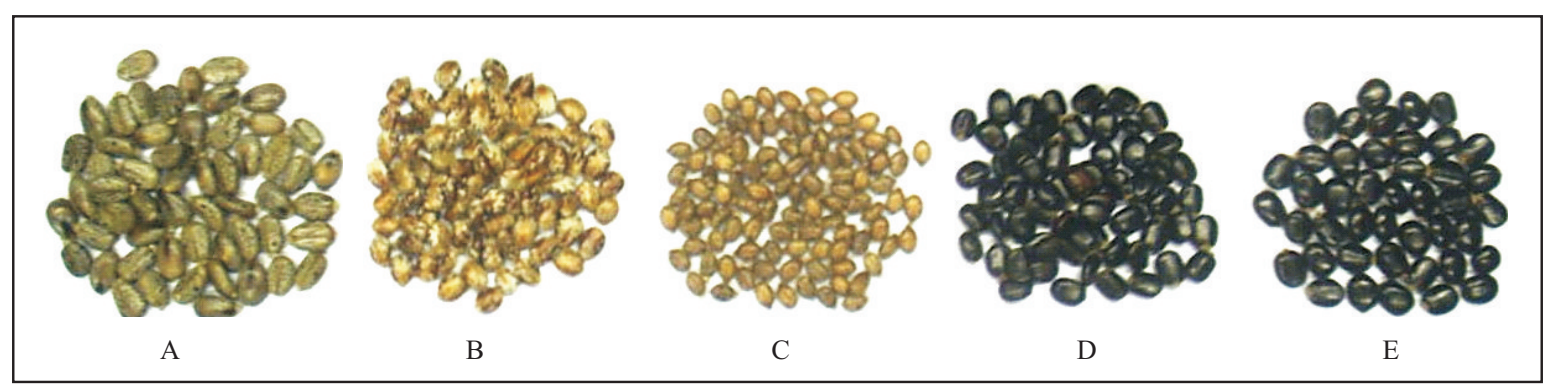

Figura 1. Variabilidade em termos de tamanho, coloração e forma das sementes utilizadas no experimento. A, Sipeal 28; B, EBDAMPA 17; C, Mirante 10; D, BRS 149 Nordestina; E, BRS 188 Paraguaçu. 
1960, pelo antigo Instituto Agropecuário do Leste (Ipeal), fator que pode ter influenciado no seu melhor desempenho. Em contrapartida, a cultivar Mirante 10 demonstrou pouca adaptação à região, visualizado em seu baixo potencial produtivo, assim como, no elevado número de racemos abortados.

O primeiro componente principal explicou $66,01 \%$ da variância total e representa um contraste entre número de racemos abortados (NRA) e o peso de sementes por racemo (PSR), peso de frutos por racemo (PFR), peso de frutos por parcela (PFP), peso de racemo (PR), uma vez que essas variáveis apresentam maiores coeficientes de ponderação, com sinais contrários (Tabela 2). O segundo componente principal explicou $20,88 \%$ e encontra-se negativamente relacionado aos caracteres comprimento efetivo do racemo (CER), comprimento do racemo (CR) e comprimento do racemo sem enchimento (CRSE).

As duas primeiras variáveis canônicas explicaram $86,89 \%$ da variação total acumulada. Este valor é considerado satisfatório, pois os componentes principais explicaram mais de $80 \%$ da variância contida no conjunto

Tabela 2. Autovetores associados a cada característica agronômica e variância total e acumulada, obtidos na análise de componentes principais de cinco genótipos de mamoneira em Cruz das Almas, BA.

\begin{tabular}{lrrrr}
\hline Características agronômicas & \multicolumn{1}{c}{ CP 1 } & \multicolumn{1}{c}{ CP 2 } & \multicolumn{1}{c}{ CP 3 } & \multicolumn{1}{c}{ CP 4 } \\
\hline FLO & $-0,2380$ & $-0,0221$ & $-0,1122$ & $-0,3535$ \\
NRE & 0,1787 & 0,3260 & $-0,0451$ & $-0,0277$ \\
NR & $-0,1655$ & 0,3392 & $-0,0561$ & $-0,1668$ \\
NRA & 0,2522 & 0,0785 & $-0,0054$ & 0,0754 \\
CER & 0,0157 & $-0,4070$ & $-0,2146$ & $-0,3396$ \\
CR & 0,0023 & $-0,4205$ & $-0,2245$ & $-0,2217$ \\
CRSE & $-0,0238$ & $-0,4270$ & $-0,2333$ & 0,0174 \\
PR & $-0,2541$ & 0,0411 & $-0,0638$ & 0,0581 \\
PFR & $-0,2550$ & 0,0455 & $-0,0350$ & $-0,0156$ \\
PSR & $-0,2555$ & 0,0387 & 0,0230 & $-0,0301$ \\
NFR & $-0,2107$ & $-0,2164$ & 0,1016 & 0,2982 \\
NSR & $-0,2156$ & $-0,2073$ & 0,0635 & 0,2988 \\
AC & $-0,2401$ & $-0,0769$ & $-0,0135$ & 0,3280 \\
AP & $-0,2142$ & 0,1100 & 0,2857 & $-0,2838$ \\
DC & $-0,2407$ & 0,0618 & $-0,1955$ & 0,1536 \\
CMIC & $-0,1156$ & $-0,2752$ & 0,4429 & $-0,1362$ \\
NIC & $-0,2010$ & 0,0731 & $-0,3177$ & 0,4059 \\
EST & $-0,1782$ & $-0,0729$ & 0,4765 & 0,1047 \\
PFP & $-0,2549$ & 0,0338 & $-0,0320$ & $-0,0823$ \\
PFPL & $-0,2163$ & 0,1067 & $-0,3273$ & $-0,0891$ \\
PSP & $-0,2489$ & 0,0651 & 0,1042 & $-0,1337$ \\
REND & $-0,2489$ & 0,0651 & 0,1042 & $-0,1337$ \\
PSPL & $-0,2263$ & 0,1516 & $-0,1929$ & $-0,1918$ \\
\hline Variância total (\%) & 66,0067 & 20,8797 & 9,2434 & 3,8702 \\
Variância acumulada (\%) & 66,0067 & 86,8865 & 96,1298 & 100,0000 \\
\hline
\end{tabular}

de caracteres analisados (Cruz, 1990; Cruz et al., 2004), viabilizando o agrupamento entre as cultivares e a construção de uma dispersão em função da diversidade observada (Figuras 2 e 3 ).

A utilização da distância $\mathrm{D}^{2}$ de Mahalanobis como medida de dissimilaridade possibilitou a formação de três grupos (Figura 2). O grupo I foi representado pelas cultivares EBDA MPA 17 e Sipeal 28; o grupo II foi composto das cultivares BRS 149 Nordestina e BRS 188 Paraguaçu; e o grupo III, pela cultivar Mirante 10.

A maior distância foi observada entre a Mirante 10 e as cultivares EBDA MPA 17 e Sipeal 28, ou seja, geneticamente a maior divergência está entre o genótipo do grupo III e os genótipos do grupo I. Portanto os melhores resultados seriam esperados da combinação entre os indivíduos desses dois grupos. Entretanto, verifica-se que a Mirante 10 apresentou baixo desempenho produtivo e pouca adaptabilidade, e segundo Abreu at al. (1999) sua utilização como genitor é desaconselhável já que as melhores combinações devem envolver indivíduos divergentes, mas de elevada performance. Resultados semelhantes foram obtidos por Costa et al. (2006).

Os melhores resultados devem ser alcançados de combinações entre os indivíduos do grupo I e os indivíduos do grupo II, por apresentarem maior distância $\mathrm{D}^{2}$ de Mahalanobis e, conseqüentemente, menor similaridade (Figura 2), além de alcançarem os maiores rendimentos e melhores desempenhos entre os componentes de produção e caracteres adaptativos (Tabela 1).

$\mathrm{Na}$ Figura 3, encontra-se a dispersão gráfica dos dados referentes aos caracteres avaliados nas seis cultivares. Os escores foram plotados em espaço

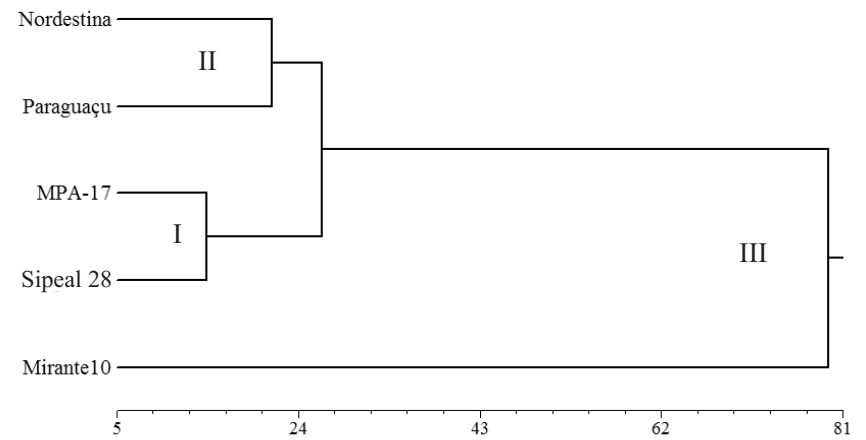

Figura 2. Dendrograma obtido a partir de 23 características agronômicas, avaliadas de cinco genótipos de mamoneira, com base na distância de Mahalanobis. 


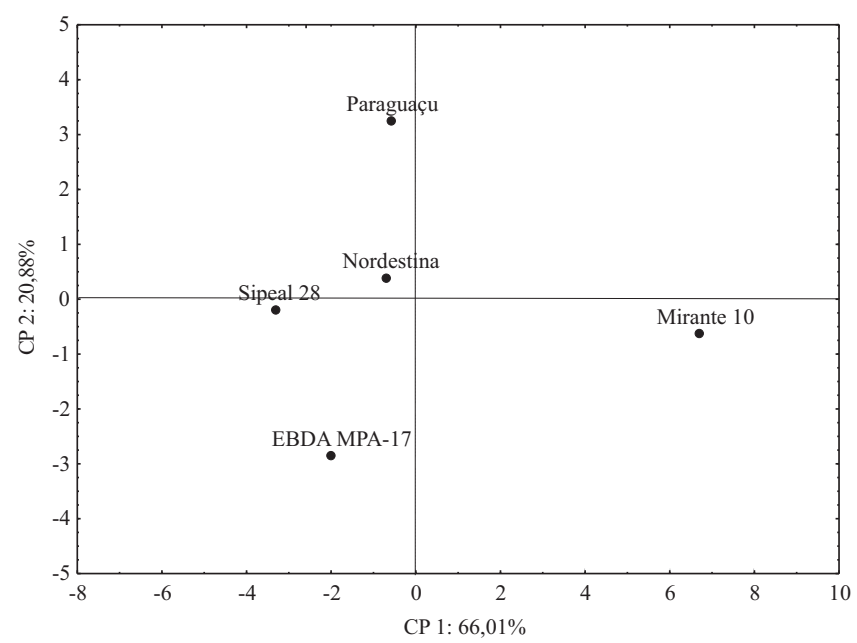

Figura 3. Dispersão gráfica dos escores de cinco genótipos de mamona em relação aos componentes principais 1 e 2 .

bidimensional, com a distância desses pontos proporcional ao grau de dissimilaridade entre os genótipos. A retenção de mais de $80 \%$ da variância total dos dados originais, pelas duas variáveis canônicas possibilitou a transposição da divergência genética do espaço p-dimensional $(\mathrm{p}=23)$ para o bidimensional, com grau de distorção desprezível, por meio da dispersão gráfica pelo método de UPGMA.

A distribuição das cultivares no gráfico de dispersão confirmou a maior distância observada entre a Mirante 10 e as demais cultivares, assim como permitiu identificar a maior divergência genética entre os indivíduos do grupo I e os do grupo II, ratificando os resultados das análises anteriores.

\section{Conclusões}

1. Há divergência genética entre as cultivares avaliadas.

2. A cultivar mais divergente, Mirante 10, não é recomendada para hibridação para formar populações segregantes, em razão de seu baixo desempenho produtivo.

3. Combinações promissoras são esperadas entre Sipeal 28 x BRS 188 Paraguaçu, Sipeal 28 x BRS 149 Nordestina, EBDA MPA 17 x BRS 188 Paraguaçu e EBDA MPA 17 x BRS 149 Nordestina, em virtude da maior dissimilaridade apresentada e do melhor desempenho médio desses genótipos.

\section{Referências}

ABREU, A. de F.B.; RAMALHO, M.A.P.; FERREIRA, D.F. Selection potential for seed yield from intra and inter-racial populations in common bean. Euphytica, v.108, p.121-127, 1999. BELTRÃO, N.E. de M. A cadeia da mamona no Brasil, com ênfase para o segmento $P \& D$ : estado da arte, demandas de pesquisa e ações necessárias para o desenvolvimento. Campina Grande: Embrapa Algodão, 2004. 20p. (Embrapa Algodão. Documentos, 129).

CARVALHO, B.C.L. Manual de cultivo da mamona. Salvador: EBDA, 2005. 65p.

COMPANHIA NACIONAL DE ABASTECIMENTO. Mamona: Brasil. Disponível em: http://www.conab.gov.br/conabweb/ download/safra/MamonaSerieHist.xls. Acesso em: 3 de abril de 2007. (Série Histórica de Área Plantada)

COSTA, M.N. da; PEREIRA, W.E.; BRUNO, R. de L.A.; FREIRE, E.C.; NÓBREGA, M.B. de M.; MILANI, M.; OLIVEIRA, A.P. de. Divergência genética entre acessos e cultivares de mamoneira por meio de estatística multivariada. Pesquisa Agropecuária Brasileira, v.41, p.1617-1622, 2006.

CRUZ, C.D. Aplicações de algumas técnicas multivariadas no melhoramento de plantas. 188p. 1990. Tese (Doutorado) - Escola Superior de Agricultura Luiz de Queiroz, Piracicaba.

CRUZ, C.D.; CARNEIRO, P.C.S. Modelos biométricos aplicados ao melhoramento genético. Viçosa: UFV, 2001. v.2. 390p.

CRUZ, C.D.; REGAZZI, A.J.; CARNEIRO, P.C.S. Modelos biométricos aplicados ao melhoramento genético. 3.ed. Viçosa: UFV, 2004. v.1. 480p.

FALCONER, D.S. Introdução à genética quantitativa. Viçosa: UFV, 1987. 279p.

FIGUEIREDO NETO, A.; ALMEIDA, F. de A.C.; GOUVEIA, J.P.G. de; NÓBREGA, M.B.M.; CARNEIRO, R.M.; PEDROZA, J.P. Divergência genética em acessos de mamona (Ricinus communis L.) baseada nas características de semente. Revista de Biologia e Ciências da Terra, v.4, p.1-10, 2004.

FREIRE, E.C.; LIMA, E.F.; ANDRADE, F.P. de. Melhoramento Genético. In: AZEVÊDO, D.M.P. de; LIMA, E.F. (Ed.). O agronegócio da mamona no Brasil. Campina Grande: Embrapa Algodão; Brasília: Embrapa Informação Tecnológica, 2001. p.229256.

RAO, A.V.; PRASAD, A.S.R.; SAI KRISHNA, T.; SECHU, D.V.; SRINIVASAN, T.E. Genetic divergence among some brown planthopper resistent rice varieties. The India Journal of Genetic Plant Breeding, v.41, p.179-185, 1981.

RIBEIRO, L.P.; SANTOS, D.M.B.; LIMA NETO, I. de A.; BARBOSA, M.F.; CUNHA, T.J.F. Levantamento detalhado dos solos, capacidade de uso e classificação de terras para irrigação da Estação de plasticultura da Universidade Federal da Bahia/Politeno em Cruz das Almas BA. Revista Brasileira de Ciência do Solo, v.19, p.105-113, 1995.

ROHLF, F.J. Numerical taxonomy and multivariate analysis system. New York: Exeter Software, 2000.

SAS INSTITUTE. System for Microsoft Windows: release 8.2. Cary: SAS, 2001. 1 CD-ROM.

$\overline{\text { Recebido em } 30 \text { de julho de } 2007 \text { e aprovado em } 25 \text { de fevereiro de } 2008}$ 